\title{
Image De-noising Method based on Wavelet Function Learning for Medical Image
}

\author{
[Sanghun Yun, Won-Seok Kang]
}

\begin{abstract}
Medical imaging is playing the key role in diagnosing and treatment of diseases. For making accurate decisions, the images acquired by various medical imaging modalities must be free from noise. So image de-noising became an important pre-processing step in Medical image analysis. In this paper, we propose a new de-noising method for medical images. Our method divides up the medical image into multiwindows and assigns the optimal mother wavelet function to each windows. And we are using an n-gram based wavelet learning technique in order to investigate optimal wavelet sequences for an image de-noising. The wavelet learning approach uses Mean Square Error (MSE) as a feature to generate an n-gram table. The performance of the proposed method is compared with the existing methods using Peak Signal to Noise Ratio (PSNR). The results showed that the proposed method has a better PSNR than the previous methods.
\end{abstract}

Keywords - Medical Image, De-noising, Wavelet Function Learning, N-gram

\section{Introduction}

Medical imaging is a technology that shows a human body and organ. It is useful to detect abnormal regions, and uses for a diagnosing and treatment of disease on the medical field. To achieve the best diagnosing, it is important that medical images be sharp and clear and without noise and artifacts. However the noise is added during acquisition of the medical images by data acquisition devices, illumination conditions and so on. Medical image enhancement techniques are important methods to increase qualities of image processing results. It often used to de-noising method in the medical image for diagnosis purposes because medical image qualities are often deteriorated by noise.

Image de-noising algorithms can be divided into spatial domain algorithm and frequency domain algorithm [1-4]. Spatial domain algorithm is to process the image pixels

directly. In the frequency domain algorithm, the image is

\author{
Sanghun Yun \\ Daegu Gyeongbuk Institute of Science \& Technology \\ Daegu, Korea
}

Won-Seok Kang

Daegu Gyeongbuk Institute of Science \& Technology

Daegu, Korea transformed from time domain to frequency domain. Then the image is processed in frequency domain and transformed to that in time domain. Mean filtering, median filtering, and adaptive Wiener filtering are classical spatial domain algorithms. It also caused some problems while removing the noise in these methods, such as serious blur of the image, especially the edge and details of the image [5]. Fourier transform is in common use in frequency domain; however, the domain characteristics of it disappeared after Fourier transform. It can neither detect the time position and the degree of intensity after signal transformation nor describe the local properties of time domain of the image [6]. The emergence and improvement of wavelet transform theory solves this problem. So wavelet has been widely used in image processing [7-8].

Many researchers has used wavelet-based techniques as a de-noise method. In previous research, wavelet de-noising methods are focused on selection of wavelet function and a threshold value (including the hard and soft threshold). In the process of image de-noising, the selection of wavelet function and the construction of threshold function are the important issues at de-noising. However previous wavelet-based method are not focused on automated optimal wavelet function selection [9-12].

In this paper, we propose a new de-noising method for medical images. Proposed method divides up the medical image into multi-windows and assigns the optimal mother wavelet function to each windows. And the method uses an ngram based wavelet learning technique in order to investigate optimal wavelet sequences for an image de-noising. The ngram approach is a type of probabilistic language model and used by the fields of computational linguistics. The wavelet learning approach uses Mean Square Error (MSE) as a feature to generate an n-gram table. Our method assigns the optimal wavelet function for each window of image and utilizes a typical wavelet de-noising method.

The rest of this paper is organized as follows: Section II describes the proposed medical image de-noise method using n-gram-based wavelet learning technique. Section III presents the experimental results to show the efficiency of the proposed method. Finally, section IV concludes the paper.

\section{Proposal De-noise Method}

In this section, we describe the detailed process of the proposed method based on wavelet transform. 
Proc. of the Second Intl. Conf. on Advances in Information Processing and Communication Technology - IPCT 2015

Copyright $\odot$ Institute of Research Engineers and Doctors, USA .All rights reserved.

ISBN: 978-1-63248-044-6 doi: 10.15224/ 978-1-63248-044-6-77

\section{A. Image Segmentation}

Let see $X$ in (1) that is the image to be analyzed. $X$ is an image that contains a noise, $\mathrm{S}$ is a noise-free image and $\mathrm{E}$ is a noise.

$$
X=S+E
$$

We divide NxM window unit of the whole image into multiwindows units to investigate the optimal mother wavelet function for each multi-window. We present the subset of $\mathrm{X}$ and in (2).

$$
x_{i j}=s_{i j}+e
$$

In (2), we can reconstruct the subsets in which the size is $\mathrm{NxM}, \mathrm{X}$ can be described as the equation as in (3).

$$
X=\begin{array}{llll}
x_{11} & x_{21} & \ldots & x_{N 1} \\
x_{12} & x_{22} & \ldots & x_{N 2} \\
\ldots & \ldots & \ldots & \ldots \\
x_{I M} & x_{2 M} & \ldots & x_{N M}
\end{array}
$$

In each divided segment $\mathrm{x}_{\mathrm{ij}}(\mathrm{i} \leq \mathrm{N}, \mathrm{j} \leq \mathrm{M})$, they have an optimum wavelet function. In the particular wavelet function as an optimal one can be assigned to the particular segment of the image. Then, we generate an optimal wavelet function series in the entire image using the optimal wavelet function that is assigned to each segment.

\section{B. Optimal Wavelet Learning}

Performance of the image de-noising based on wavelet transform is determined by the selection of the wavelet function. The problem is how to choose the best wavelet function. In order to find the optimal wavelet function, we adopt the probabilistic approach, called n-gram.

The wavelet function learning for our method is performed in the noisy-free image only. We divide the input image into NxM of multi windows. There are more than 50 mother wavelet functions such as Daubechies, Symlets, Coiflets, Biorthogonal, Reverse biorthogonal, Discrete approximation of Meyer family. We can handle DWT with above mother wavelet function. The number of mother wavelet function is $\mathrm{L}$.

We apply a forward and inverse DWT function to each segmented image $\left(\mathrm{x}_{\mathrm{ij}}\right)$. Then, we calculate a Mean Square Error (MSE) between the before DWT function applying $\left(\mathrm{x}_{\mathrm{ij}}\right)$ and after DWT function applying $\left(\hat{\mathrm{x}}_{\mathrm{ij}}\right)$ as in (4) for segmented image.

$$
M S E_{l i j}=\frac{I}{M * N} \sum_{i=1}^{L} \sum_{i=1}^{N} \sum_{j=1}^{M}\left[x_{i j}(l)-\dot{x}_{i j}(l)\right]^{2}
$$

Where $x_{i, j}$ is a subset of multi-windows which is a part of the noise-free image, and $\hat{x}_{i, j}$ is the reconstructed image of $x_{i, j}$ by the inverse DWT using l-th wavelet function $(1 \leq \mathrm{L}, \mathrm{i} \leq \mathrm{N}, \mathrm{j}$ $\leq \mathrm{M})$. $\mathrm{MSE}_{\mathrm{lij}}$ is used in evaluate the restoration quality of the reconstructed image. If a result of $\mathrm{MSE}_{\mathrm{lij}}$ is closer to 0 , it can be one of the best candidates. Table. 1 shows an example of calculating $\mathrm{MSE}_{\mathrm{lij}}$ for each segmentation image. The row is the index of the separated image $\left(\mathrm{x}_{\mathrm{ij}}\right)$ and the column is the index of the particular mother wavelet functions.

\section{TABLE I. EXAMPLE OF THE MSE MATRIX $_{L}$ MATR}

\begin{tabular}{|c|c|c|c|c|}
\hline \multirow{2}{*}{ Wavelet } & \multicolumn{4}{|c|}{ Image } \\
\cline { 2 - 5 } & $x_{11}$ & $x_{12}$ & $\cdots$ & $x_{N M}$ \\
\hline $\mathrm{db} 1$ & 0.0192974825 & 0.0087794476 & $\cdots$ & 0.0081033218 \\
\hline $\mathrm{db} 2$ & 0.0163827631 & 0.0085858928 & $\cdots$ & 0.0065050650 \\
\hline $\mathrm{db} 3$ & 0.0173858875 & 0.0084810555 & $\cdots$ & 0.0069246949 \\
\hline $\mathrm{db} 4$ & 0.0178079725 & 0.0081695489 & $\cdots$ & 0.0067570046 \\
\hline$\ldots$ & $\ldots$ & $\ldots$ & $\cdots$ & $\ldots$ \\
\hline
\end{tabular}

In the next step, we transform the $\mathrm{MSE}_{\mathrm{lij}}$ matrix into the frequency-based table to make an n-gram table. We use a heuristic method applying the threshold rule like (5) to convert the $\mathrm{MSE}_{\mathrm{lij}}$ matrix.

$$
\begin{aligned}
& \operatorname{ferq}\left(M S E_{i j}\right)= \\
& \left\{\begin{array}{l}
1, \text { if } M S E_{i j} \\
0, \text { otherwise }
\end{array}\right.
\end{aligned}
$$

Where $\mathrm{MSE}_{\mathrm{ij}}$ denotes the whole elements of the particular column. If each cell of the $\mathrm{MSE}_{\mathrm{lij}}$ matrix satisfies the rule, the frequency of each cell is accumulatively increased by 1 . If not, the frequency does not change. Table 2 shows an example of the $\mathrm{MSE}_{\mathrm{lij}}$ frequency matrix for all cells. The more it repeats the wavelet learning, the more the probabilistic value becomes more precise because it does not reset the frequency. It is meaningful that the proposed method can generate the probabilistic table for each data acquisition environment.

\section{TABLE II. EXAMPLE OF THE MSE LIJ $_{\text {FREQUENCY MATRIX }}$}

\begin{tabular}{|c|c|c|c|c|c|}
\hline \multirow{2}{*}{ Wavelet } & \multicolumn{5}{|c|}{ Image } \\
\cline { 2 - 6 } & $\cdots$ & $x_{18}$ & $x_{19}$ & $\cdots$ & $x_{N, M}$ \\
\hline $\mathrm{db} 1$ & $\cdots$ & 1 & 1 & $\cdots$ & 1 \\
\hline $\mathrm{db} 2$ & $\cdots$ & 1 & 1 & $\cdots$ & 0 \\
\hline $\mathrm{db} 3$ & $\ldots$ & 1 & 0 & $\cdots$ & 0 \\
\hline $\mathrm{db} 4$ & $\ldots$ & 1 & 0 & $\cdots$ & 0 \\
\hline$\ldots$ & $\ldots$ & $\ldots$ & $\ldots$ & $\cdots$ & $\ldots$ \\
\hline
\end{tabular}

An n-gram is used in predicting the next item when given the previous items. An n-gram of size 1 is referred as uni-gram. An n-gram of size 2 is bi-gram, and size 3 is a tri-gram. An n- 
gram can infer the next item while estimating the probability function $\mathrm{P}$ as in (6).

$$
P\left(s_{n} \mid s_{l}, s_{2}, \ldots, s_{n-1}\right)=\frac{P\left(s_{l}, \ldots, s_{n}\right)}{P\left(s_{l}, \ldots, s_{n-1}\right)}
$$

Where $\mathrm{s}$ is a symbol item proposing to predict, $\mathrm{n}$ is an index number in symbol series. The probability of the sequential symbol can be transformed into the frequency-based probability as following (7).

$$
P\left(s_{n} \mid s_{1}, s_{1}, \ldots, s_{n-1}\right)=\frac{\operatorname{freq}\left(s_{1}, \ldots, s_{n}\right)}{\operatorname{freq}\left(s_{l}, \ldots, s_{n-1}\right)}
$$

Where freq $\left(\mathrm{s}_{1}, \ldots, \mathrm{s}_{\mathrm{n}}\right)$ is a frequency value according to the Markov assumption. In this paper, we use the $\mathrm{MSE}_{\mathrm{lij}}$ frequency matrix to produce the n-gram table. We use a bigram table to lookup into as the best optimal wavelet function. A bi-gram table is consist of a pair of terminal symbol. The first symbol is previous item of the index, and the second symbol is current item of the index in the sequence. In the bigram table, terminal symbol is a name of the wavelet function. Table 3 shows an example of the bi-gram table. It is used in de-noising module later.

TABLE III. EXAMPLE OF THE BI-GRAM TABLE

\begin{tabular}{|c|c|}
\hline \multicolumn{2}{|c|}{ Bi-gram Table } \\
\hline $\begin{array}{c}\text { A pair of } \\
\text { Wavelet Function }\end{array}$ & value \\
\hline$\ldots$ & $\ldots$ \\
\hline $\mathrm{db} 3-\mathrm{db} 4$ & 0.0157853197 \\
\hline $\mathrm{db} 3-\mathrm{db} 5$ & 0.0263088661 \\
\hline $\mathrm{db} 3-\mathrm{db} 6$ & 0.0265719547 \\
\hline $\mathrm{db} 3-\mathrm{db} 7$ & 0.0252565114 \\
\hline$\ldots$ & $\ldots$ \\
\hline
\end{tabular}

The wavelet function learning procedure is illustrated as Figure 1. The wavelet learning module generates a bi-gram table.

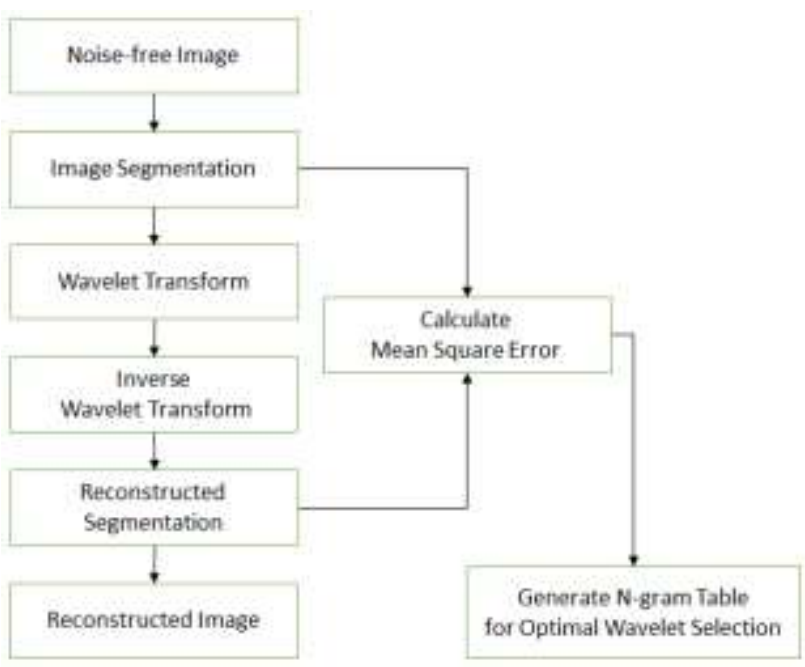

Figure 1. The wavelet function learning procedure.

\section{De-noising with $\mathbf{N}$-gram Table}

The proposed de-noising procedure based on the probability of bi-gram table is as Figure 2. The steps are as follows:

Step. 1: Devide a noisy image into the unit of the predefined segment size: $N x M$.

Step. 2: Find the wavelet function name with the maximum probability. Assign to the first mother wavelet function. Then, make the de-noising segment through inverse DWT by soft thresholding which was proposed by Donoho [11].

Step. 3: Supposed that the previous terminal symbol is assigned at Step 2. Searching for the first terminal symbol in the bi-gram table, then find the second terminal symbol with the maximum probability.

Step. 4: Repeat Step 2 and Step 3, until performed for all segments

Step. 5: Reconstruct the de-noised image by merge the respective de-noised segments.

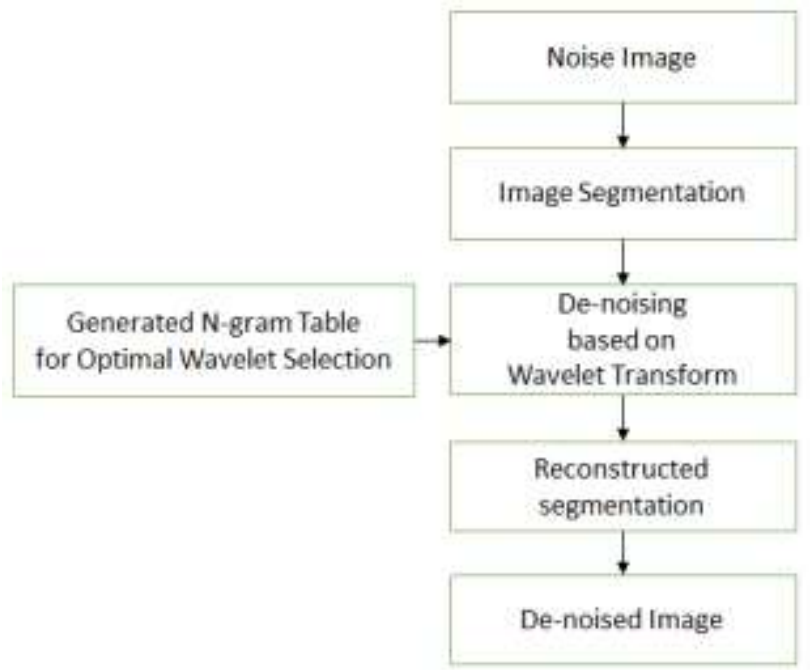

Figure 2. The de-noising procedure with n-gram table. 


\section{Experimental Results}

In order to confirm the superiority of the proposed method was tested as the following. We tested some of the eardrum image taken by endoscopy. The aim of our comparison is to point out the differences in term of PSNR (Peak Signal to Noise Ratio) due to a particular choice of wavelet bases. The PSNR has been computed using the following formula.

$$
\begin{aligned}
\text { PSNR } & =10 \cdot \log _{10}\left(\frac{M A X_{l}^{2}}{M S E_{l}}\right) \\
& =20 \cdot \log _{10}\left(M_{l}\right)-10 \cdot \log _{10}\left(M_{l} E_{l}\right)
\end{aligned}
$$

Where $\mathrm{MAX}_{\mathrm{I}}$ is the maximum possible pixel value of the image. $\mathrm{MSE}_{\mathrm{I}}$ is the sum over all squared value differences divided by image size. It denoted as (9), (10).

$$
\begin{gathered}
M A X_{I}=2^{H}-1 \\
M S E_{l}=\frac{1}{N \cdot M} \sum_{i=1}^{N} \sum_{j=1}^{M}[X(i, j)-\hat{X}(i, j)]^{2}
\end{gathered}
$$

We have set up experimental parameters as follows: the number of segments size in learning procedure is $64 \times 64$, the number of segments size in testing procedure is from $1 \times 1$ to $30 \times 30$, the PSNR of noisy image is $70.7 \mathrm{~dB}$, noise type is salt and pepper, the wavelet decomposition level is 5 .

Figure 3 show the change trend of PSNR according to the number of segment. The best PSNR is $87.5 \mathrm{~dB}$ at $23 \times 23$ segments. The proposed method has been de-noised over the $23.8 \%$ than before. When selecting one wavelet $(\mathrm{db} 3)$ for denoising, a PSNR of the image de-noising is $72.5 \mathrm{~dB}$. The improvements of PSNR is $2.5 \%$. Our method showed a performance improvement of $20.7 \%$ using one wavelet function (db3). Figure 4 shows the results of the image denoising.

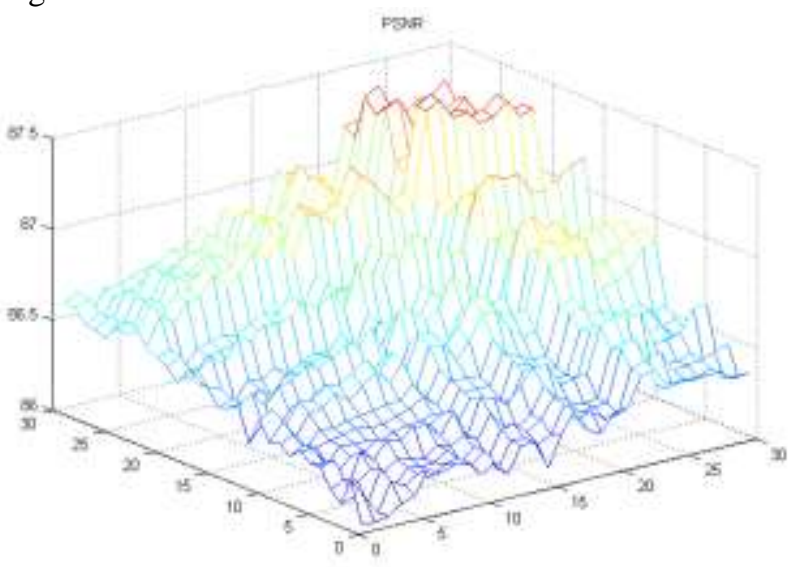

Figure 3. Change trend of PSNR according to the number of segment.

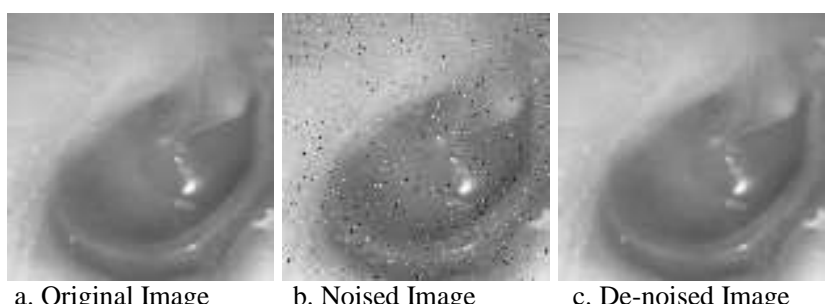

Figure 4. Result of De-noised Image.

\section{Conclusions}

Medical image de-noising techniques are important way to improve the quality of the image processing result. For accurate diagnosis, we need a variety noise removal. The wavelet transform has been used effectively in the noise removal. In this paper, we propose a new de-noising method for medical images. First, our proposed method divides the medical image into NxM segments, and assigns the optimal wavelet functions in each segment. Second, we use n-gram based wavelet learning techniques to find the best order of the wavelet sequences for an image de-noising. The wavelet learning approach uses Mean Square Error (MSE) as a feature to generate an $\mathrm{n}$-gram table. $\mathrm{N}$-gram table is used in denoising module later. Finally, proposed method performs the optimum noise elimination by lookup to the bi-gram table. The performance of the proposed method is compared with the existing methods using Peak Signal to Noise Ratio (PSNR). Experimental results demonstrate that the proposed method is provided an improved PSNR than previous methods.

\section{Acknowledgment}

This work was supported by the SME Convergence Technology Development Program of the Small and Medium Business Administration of Korea (S2098563).

\section{References}

[1] Z. Jia, S. Hui, D. Cheng-Zhi, and C. Xi, "Particle swarm optimization based adaptive image denoising in shearlet domain , Journal of Chinese Computer Systems, vol. 32, no. 6, pp. 1147-1150, 2011.

[2] N.-F. Yang, C.-M. Wu, and H.-Z. Qu, "Mixed noised denoised based on partial differential equation," Application Research of Computers, vol. 30, no. 6, pp. 1899-1902, 2013.

[3] Y. Zhang, P. Zhang, G.Wang, andH. Zhuo, "Denoisingmethod for color images based on chrominance model and curvelet transform," Journal of Image and Graphics, vol. 17, no. 12, pp. 1472-1477, 2012.

[4] L. Zhang and X. Li, "Image denoising method of partial differential equation based on wavelet transform," Laser \& Infrared, vol. 43, no. 8, pp. 943-946, 2013.

[5] J.Yang, C.Wu, andH. Qu, "Multi-parameter threshold function for image de-noising based on wavelet transform," Computer Engineering and Applications, vol. 48, no. 13, pp. 176-180, 2012.

[6] Q. Wang, L.-Y. Gong, W.-J. Ren, F.-C. Huo, and F. Gao, “The algorithm of image denoising based on the improved method of 
Proc. of the Second Intl. Conf. on Advances in Information Processing and Communication Technology - IPCT 2015

Copyright $\odot$ Institute of Research Engineers and Doctors, USA .All rights reserved.

ISBN: 978-1-63248-044-6 doi: 10.15224/ 978-1-63248-044-6-77

wavelet thresholding," Techniques of Automation \& Applications, vol. 32, no. 11, pp. 61-66, 2013.

[7] X.U. Jing, F.U.Wei,H.U. Cai-Ning, andW.U.Hua, "Implementation of improved embedded Zerotree wavelet image coding algorithm," Radio Communications Technology, vol. 34, no. 5, pp. 62-64, 2008.

[8] L. Zhai and L. Zhao, "Adaptive image watermarking algorithm based on wavelet packet transform," Radio Engineering, vol. 43, no. 2, pp. 23-26, 2013.

[9] S. Mallat and S. Zhong, "Characterizating of singnal from multiscale edges," IEEE Transactions on Pattern Analysis and Machine Intelligence, vol. 14, no. 7, pp. 710-732, 1992.

[10] H. X. Yang,X. S.Wang, P.H. Xie, A. Leng, andY. Peng, "Infrared image denoising based on improved threshold and inter-scale correlations of wavelet transform," Acta Automatica Sinica, vol. 37, no. 10, pp. 1167-1174, 2011.

[11] D. L. Donoho, "De-noising by soft-thresholding," IEEE Transactions on Information Theory, vol. 41, no. 3, pp. 613627, 1995.

[12] M.U. Juan, D. U. Chao-Ben, andY. I. Zhou, "Adaptive threshold for remote sensing image denoising based on wavelet and NSCT," Radio Engineering, vol. 42, no. 11, pp. 23-25, 2012.

About Author (s):

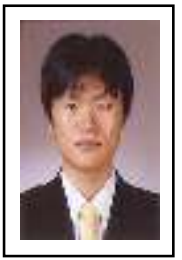

Sanghun Yun
Researcher, in Daegu Gyeongbuk Institute
of Science \& Technology
Interested in Wireless Sensor Network,
Image Processing, Signal Processing,
Machine Learning

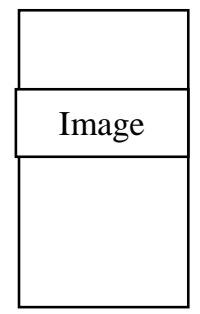

Won-Seok Kang

Senior Researcher, in Daegu Gyeongbuk Institute of Science \& Technology Interested in EEG, Brain Dynamics, Signal Analysis, Parallel Modeling, Wavelet Analysis 